\section{Conclusion et perspectives}

Définir la nature des stimulus, infectieux ou tissulaires, qui coopèrent avec les mutations de 3BP2 pour déclencher localement l'auto-inflammation pathologique à l'origine du chérubisme permettra de mieux comprendre les mécanismes moléculaires et cellulaires à l'origine de cette maladie génétique rare, et, en particulier, d'identifier précisément les multiples voies de signalisation mises en jeu. Le ciblage pharmacologique de ces voies représente clairement une stratégie thérapeutique prometteuse, comme l'atteste une étude récente [10]. Alternativement, il sera également intéressant de déterminer si le traitement des infections buccales chez le jeune patient atteint de chérubisme peut atténuer le développement de la maladie. $\diamond$
SH3BP2 heterozygous mutation amplifies macrophage inflammatory responses to infection in a mouse model of cherubism

\section{LIENS D'INTÉRÊT}

Les auteurs déclarent n'avoir aucun lien d'intérêt concernant les données publiées dans cet article.

\section{RÉFÉRENCES}

1. Reichenberger $\varepsilon$ J, Levine MA, Olsen BR, et al. The role of SH3BP2 in the pathophysiology of cherubism. Orphanet J Rare Dis 2012 ; 7 : S5.

2. Deckert M, Tartare-Deckert S, Hernandez J, et al. Adaptor function for the Syk kinases-interacting protein 3BP2 in IL-2 gene activation. Immunity 1998 ; $9:$ 595-605.

3. Deckert M. L'adaptateur 3BP2. Quelle place pour cette protéine dans la signalisation des leucocytes? Med Sci (Paris) 2006; 22 : 1081-6.

4. GuezGuez A, Prod'homme V, Mouska X, et al. 3BP2 Adapter protein is required for receptor activator of NFkappaB ligand (RANKL)-induced osteoclast differentiation of RAW264.7 cells. J Biol Chem 2010 ; $285: 20952-63$.
5. Ueki Y, Lin Cy, Senoo M, et al. Increased myeloid cell responses to $\mathrm{M}$-CSF and RANKL cause bone loss and inflammation in SH3BP2 cherubism mice. Cell 2007 ; 128: 71-83.

6. Prod'Homme V, Boyer L, Dubois N, et al. Cherubism allele heterozygosity amplifies microbe-induced inflammatory responses in murine macrophages. J Clin Invest $2015 ; 125$ : 1396-400.

7. Levaot N, Voytyuk 0 , Dimitriou I, et al. Loss of Tankyrase-mediated destruction of 3BP2 is the underlying pathogenic mechanism of cherubism. Cell $2011 ; 147$ : 1324-39.

8. Graves DT, Li J, Cochran DL. Inflammation and uncoupling as mechanisms of periodontal bone loss. J Dent Res 2011 ; 90 : 143-53.

9. Yoshitaka T, Mukai T, Kittaka M, et al. Enhanced TLR-MYD88 signaling stimulates autoinflammation in SH3BP2 cherubism mice and defines the etiology of cherubism. Cell Rep $2014 ; 8$ : 1752-66.

10. Kadlub N, Vazquez MP, Galmiche L, et al. The calcineurin inhibitor tacrolimus as a new therapy in severe cherubism. J Bone Miner Res 2014 ; 30 : 878-85.

\title{
NOUVELLE
}

\section{L'épithélium thymique, un passé dans la dualité et un présent unifié}

Victoria Michaels Lopez, Sophie Ezine
> Pour assurer une thymopoïèse complète aboutissant au développement de lymphocytes $T$ fonctionnels, un stroma thymique mature est requis. Récemment, deux articles nous dévoilent le «passé » surprenant de cet organe, ainsi que la source cellulaire à l'origine de l'hétérogénéité de son épithélium $[1,2]$. L'ensemble de ces données améliorent nos connaissances des pathologies thymiques et permettront peut-être de proposer de nouvelles stratégies de thérapie cellulaire visant à contrer l'involution thymique et ses conséquences.
Les cellules épithéliales thymiques: des régulateurs essentiels du développement et de la sélection des lymphocytes $\mathrm{T}$

Le développement et la maturation des lymphocytes $T$ sont uniques par rapport aux autres lignées hématopoïétiques, car ils prennent place dans un organe lymphoïde particulier, le thymus. Dans les différentes niches thymiques, les progéniteurs hématopoïétiques, en provenance du foie fœtal et/ou de la moelle osseuse, se différencient principalement en cellules $T$ porteuses d'un répertoire diversifié de récepteurs spécifiques pour
Université Paris Descartes - site Broussais CS61431, 14, rue Maria Helena Vieira Da Silva, batiment Leriche-porte 14, pièce L-507, 75993 Paris Cedex 14, France.

\section{sophie.ezine@inserm.fr}

différents antigènes nommés TCR. Ce développement est guidé par les cellules stromales thymiques, qui ont pour principal composant les cellules épithéliales thymiques. Les cellules épithéliales thymiques forment un microenvironnement fonctionnel spécialisé dans le maintien des étapes de la maturation T. Elles fournissent tous les éléments nécessaires (cytokines, chimiokines, ligands) au contrôle de l'acquisition d'une spécificité lymphocytaire $T$, de la migration, de la survie, de la prolifération et de la sélection de ces lymphocytes [3].

Le thymus est composé de lobes (deux chez la souris, plusieurs lobules chez 


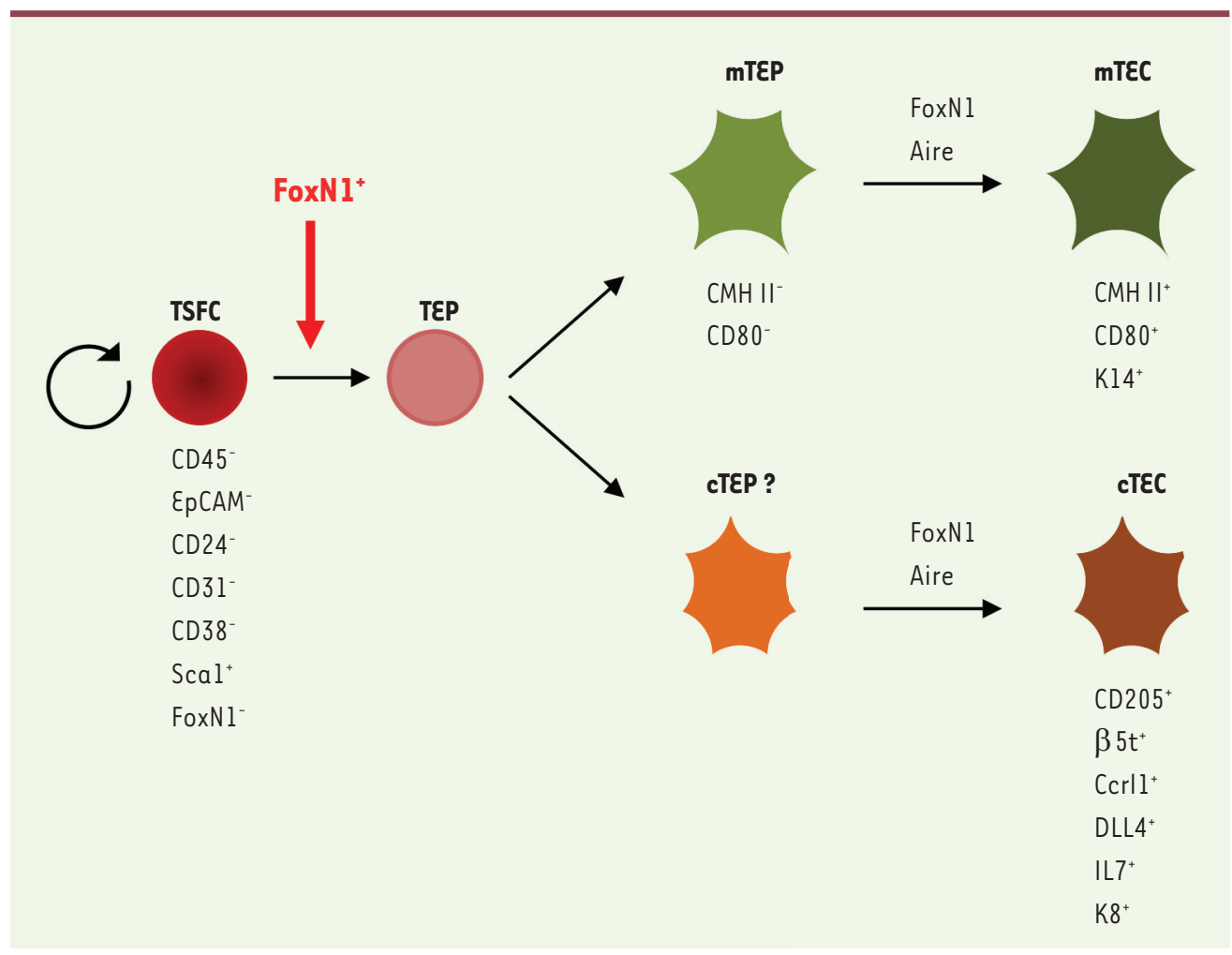

Figure 1. Modèle du développement de l'épithélium thymique chez la souris adulte. La cellule souche thymosphere-forming cell (TSFC) se forme indépendamment du facteur FoxNl. En revanche, sa différenciation en progéniteurs bipotents de l'épithélium thymique (TEP) et la maturation des cellules épithéliales thymiques matures corticales (cTEC) et médullaires (mTEC) requièrent FoxN1.

sevrage. Après la puberté, il involue lentement selon un processus non élucidé. Lors de ces premières étapes, l'expression du facteur de transcription forkhead 1 (FoxNl) dans

I'homme) entourés d'un tissu conjonctif. Chaque lobe est constitué d'une partie centrale médullaire formée de cellules épithéliales thymiques médullaires (mTEC), et d'une partie périphérique corticale composée de cellules épithéliales thymiques corticales (cTEC). Ces deux compartiments possèdent des caractéristiques phénotypiques et fonctionnelles distinctes [3]. Les cT\&C ont un rôle important lors des étapes primaires du développement $T$, elles permettent l'engagement et la prolifération des précurseurs T via deux molécules cruciales à ce stade : l'expression du ligand de Notchl delta like 4 (DL4) et de la cytokine interleukine (IL)-7 [4]. Le cortex permet aussi la sélection des thymocytes double-positifs (coexprimant les molécules CD4 et CD8) notamment par l'expression d'une diversité de peptides du «soi » présentés par les molécules du CMH (complexe majeur d'histocompatibilité) de classes I et II. Dans la partie médullaire du thymus, les mTEC jouent, avec les cellules dendritiques très probablement produites in situ, un rôle décisif dans les dernières étapes du développement T. Elles permettent la sélection négative des cellules $T$ autoréactives (susceptibles de réagir contre les protéines du soi) et la génération des cellules T régulatrices [3].

Malgré leurs différences fonctionnelle et anatomique, les cTEC et mTEC partagent quelques marqueurs phénotypiques comme par exemple l'expression de la molécule d'adhésion CD326 (EpCAM, epithelial cell adhesion molecule) et le CMH II. Elles n'expriment pas la molécule CD45 spécifique des cellules hématopoïétiques, et sont donc classées comme non hématopoïétiques [4].

\section{Origine des cellules épithéliales} thymiques dans le thymus adulte?

Le thymus commence à se développer autour de 10,5 jours de gestation chez la souris ( 8 à 10 semaines de gestation chez l'homme). La première colonisation hématopoïétique se produit autour de $\varepsilon 11,5$, et la délimitation des compartiments du cortex et de la médulla est reconnaissable à partir de $\varepsilon 14$ [4]. À partir de ce stade chez la souris, la taille du thymus augmente et ce jusqu'au les progéniteurs épithéliaux thymiques est indispensable au développement du thymus, et, ensuite, à la génération et au maintien des fonctions des $\mathrm{mTEC}$ et cTદC matures [4].

Les mécanismes du maintien des cellules épithéliales thymiques dans le thymus adulte sont mal élucidés à ce jour. Si l'hétérogénéité du compartiment épithélial ne fait pas de doute, son ontogenèse est difficile à comprendre, comme le soulignent plusieurs travaux. Il a été suggéré que le cortex se développe à partir d'une cellule souche d'origine ectodermique tandis que la médulla dérive de l'endoderme [5]. D'autres travaux suggèrent que les précurseurs de ces deux types cellulaires ont pour origine le seul endoderme [6]. Les travaux de Gill et al., en Australie, ont permis une avancée significative en identifiant un progéniteur épithélial fœtal, exprimant la glycoprotéine MTS24, capable de générer les lignées corticale et médullaire [7]. Bleul et al. ont également caractérisé, chez la souris adulte, un progéniteur épithélial thymique bipotent, équivalent au progéni- 


\begin{tabular}{lccccc}
\hline & $\begin{array}{c}\text { Expression de } \\
\text { FoxN4 }\end{array}$ & $\begin{array}{c}\text { Expression de } \\
\text { FoxN1 }\end{array}$ & $\begin{array}{c}\text { Expression de } \\
\text { DL4 }\end{array}$ & $\begin{array}{c}\text { Expression de } \\
\text { I’IL-7 }\end{array}$ & $\begin{array}{c}\text { Spécificité } \\
\text { thymique }\end{array}$ \\
$\begin{array}{l}\text { Lamproie (vertébrés } \\
\text { sans mâchoire) }\end{array}$ & + & - & Faible & Torte & et B \\
\hline Souris & - & + & Forte & Forte & $T$ \\
\hline
\end{tabular}

Tableau I. Évolution de la spécificité thymique chez la lamproie et la souris. Expression des facteurs de transcription FoxNl et FoxN4, du ligand DL4 et de la cytokine IL7 dans l'épithélium thymique. (+) exprimé ; (-) absent.

teur fœtal décrit par Gill [8]. Pourtant, la caractérisation et la purification de ces progéniteurs épithéliaux thymiques bipotents ont jusqu'ici rencontré un succès limité et leur phénotype complet reste encore à définir.

Un travail récent original, basé sur une méthode atypique de culture, a permis d'observer la formation de sphères à partir de cellules stromales thymiques adultes [1]. Ces «thymosphères » proviennent de la lente division d'une cellule, la thymosphere-forming cell. La dissociation des sphères «primaires » permet de reformer des sphères «secondaires ». La présence de précurseurs des mTEC et cTEC dans ces thymosphères a été évaluée in vitro et in vivo. In vitro, les celIules épithéliales thymiques matures, qui expriment les kératines 14 ( $\left.\mathrm{K} 14^{+}, \mathrm{mTEC}\right)$ et $8\left(K 8^{+}, c T E C\right)$, sont identifiées 9 jours après culture. In vivo, la capacité des thymosphere-forming cells à générer des cellules épithéliales thymiques matures a été démontrée après leur greffe sous la capsule rénale de souris receveuses athymiques (nude). Ces cellules formant des sphères peuvent donc être qualifiées de cellules souches de l'épithélium thymique. Elles sont présentes dans le thymus jeune, mais aussi dans le thymus en involution. Elles se forment aussi en culture à partir de thymus humain. Si
FoxNl n'est pas requis pour leur génération, en revanche il est nécessaire à leur différenciation en cellules épithéliales thymiques matures (Figure 1).

\section{La bifonctionnalité thymique ancestrale}

Le gène Foxnl s'est formé par duplication d'un gène ancestral Foxn4 présent chez le poisson, mais pas chez la souris [9]. Une étude de Swann et al. [2] montre que l'introduction de FoxNI chez la souris nude (porteuse d'une mutation spontanée du gène Foxnl) permet de restaurer le développement T, alors que celle de FoxN4 aboutit au développement de cellules $B$ dans le thymus. Dans ces conditions, c'est la diminution de DL4 (dont l'expression dépend de FoxN1) et le maintien de la production d'IL-7 qui promeuvent le développement $B$ à l'identique de ce qui est connu dans la moelle osseuse. Ces travaux soulignent le rôle dual du thymus primordial, qui est compétent pour assurer la lymphopoï̀se aussi bien T que B. La question est donc maintenant de comprendre quels mécanismes survenus au cours de l'évolution sont responsables de la perte, par le thymus, de la capacité de promouvoir le développement des cellules B (Tableau I).

L'ensemble de ces données devraient permettre d'envisager sous un angle nouveau le traitement des pathologies associées à un thymus dysfonctionnel, qu'il s'agisse de pathologies autoimmunes ou dans le cadre de décifits immunitaires. $\diamond$

Thymic epithelial populations: recently reunified through a unique stem cell

\section{LIENS D'INTÉRÊT}

Les auteurs déclarent n'avoir aucun lien d'intérêt concernant les données publiées dans cet article.

\section{RÉFÉRENCES}

1. Ucar A, Ucar O, Klug P, et al. Adult thymus contains FoxN1- epithelial stem cells that are bipotent for medullary and cortical thymic epithelial lineages. Immunity $2014 ; 41: 257-69$.

2. Swann JB, Weyn A, Nagakubo D, et al. Conversion of the thymus into a bipotent lymphoid organ by replacement of Foxnl with its paralog, Foxn4. Cell Rep $2014 ; 8$ : 1184-97.

3. Takahama Y. Journey through the thymus: stromal guides for T-cell development and selection. Nat Rev Immunol 2006 ; 6 : 127-35.

4. Rodewald HR. Thymus organogenesis. Annu Rev Immunol 2008 ; 26 : 355-88.

5. Cordier AC, Haumont SM. Development of thymus, parathyroids, and ultimo-branchial bodies in NMRI and nude mice. Am J Anat $1980 ; 157: 227-63$.

6. Le Douarin NM, Jotereau FV. Tracing of cells of the avian thymus through embryonic life in interspecific chimeras. J Exp Med 1975 ; 142 : 17-40.

7. Gill J, Malin M, Holländer GA, Boyd R. Generation of a complete thymic microenvironment by MTS $24^{+}$thymic epithelial cells. Nat Immunol 2002 ; 3 : 635-42.

8. Bleul CC, Corbeaux T, Reuter A, et al. Formation of a functional thymus initiated by a postnatal epithelial progenitor cell. Nature 2006 ; 441 : 992-6.

9. Bajoghli B, Aghaallaei N, Hess I, et al. Evolution of genetic networks underlying the emergence of thymopoiesis in vertebrates. Cell $2009 ; 138: 186-97$

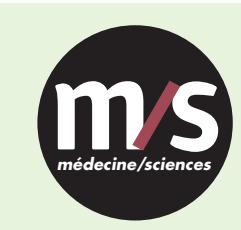

Tarifs d'abonnement $\mathrm{m} / \mathrm{s}-2015$

Abonnez-vous

à médecine/sciences
$>$ Grâce à $\mathrm{m} / \mathrm{s}$, vivez en direct les progrès des sciences biologiques et médicales

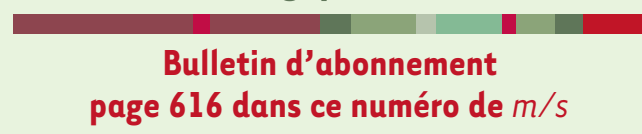

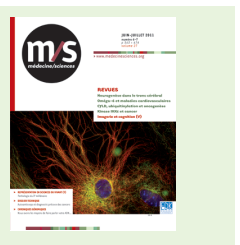

